\title{
Imunoterapia com aeroalergénios em tempos de COVID-19
}

\section{Aeroallergen immunotherapy during COVID-I 9 pandemic}

Data de receção / Received in: 4/10/2020 Data de aceitação / Accepted for publication in: 3/1/2021

\author{
Rev Port Imunoalergologia 2021;29(3): 179-196
}

\begin{abstract}
Joana Cosme' iD, Amélia Spínola Santos' iD, Anabela Resende², Susana Marques², Elisa Pedro' iD
' Serviço de Imunoalergologia, Hospital de Santa Maria, Centro Hospitalar Universitário de Lisboa Norte, Lisboa, Portugal

${ }^{2}$ Centro de Ambulatório, Consulta Externas, Centro Hospitalar Universitário de Lisboa Norte, Lisboa, Portugal
\end{abstract}

Este trabalho original recebeu o 2. ${ }^{\circ}$ prémio SPAIC-Roxall 2020.

\section{RESUMO}

Fundamentos: A imunoterapia subcutânea a aeroalergénios (ITASC) foi suspensa em alguns serviços durante o confinamento em contexto da pandemia por COVID-19. Objetivos: Avaliar o impacto da suspensão da ITASC nos sintomas, necessidade de medicação de controlo e qualidade de vida e, ainda, conhecer as expectativas e grau de segurança/receio dos doentes em voltar ao hospital. Métodos: Inquérito anónimo para autopreenchimento pelos doentes com mais de 12 anos nas primeiras 5 semanas após reinício da ITASC. Utilizou-se o CARAT como instrumento de avaliação do controlo dos sintomas de rinite e asma. Resultados: Incluíram-se 77 doentes ( $90 \%$ adultos, $68 \%$ mulheres), todos com rinite e $40 \%$ com asma. $O$ intervalo de tempo médio entre a última administração e o reinício da ITASC foi de $13 \pm 2,48$ semanas. Foram observados em consulta urgente/não programada por agudização da patologia respiratória $7 \%$ dos doentes e apenas I teve COVID-19. As pontuações CARAT-Total revelaram controlo dos sintomas de rinite e de asma em $35 \%$ e $66 \%$ dos doentes, respetivamente. A proporção de doentes não controlados aumentou com o alargamento do período de suspensão (PS) da ITASC. A maioria (>90\%) dos doentes referiu não haver impacto significativo na sua qualidade de vida e manteve a medicação de controlo habitual (48\%), referiu preocupar-se em perder os benefícios da ITASC com a sua suspensão (62\%) e referiu sentir-se mais seguro nos gabinetes de vacinas do que no edifício do hospital ou sala de espera. Conclusões: Registou-se um aumento da frequência de doentes não controlados com o aumento do PS. Para a maioria, o PS não teve impacto significativo na qualidade de vida. A frequência de doentes que necessitaram de ser observados em consulta urgente/não programada foi 
baixa e apenas I referiu ter tido COVID-19. A perceção de segurança na sala de vacinas é maior do que nos espaços comuns do hospital.

Palavras-chave: Asma, CARAT, COVID-19, imunoterapia subcutânea a alergénios, medicação, sintomas, qualidade de vida, rinite.

(C) 2021 Sociedade Portuguesa de Alergologia e Imunologia Clínica. Publicado por Publicações Ciência \& Vida. Este é um artigo Open Access sob uma licença CC BY-NC-ND (http://creativecommons.org/licenses/by-nc-nd/4.0/)

\section{ABSTRACT}

Background: Subcutaneous immunotherapy with aeroallergen (SCITA) was stopped in some departments during the lockdown period due to the COVID-19 pandemic. Objectives: To evaluate the impact of SCITA interruption on symptoms, the need for control medication, the quality of life, and the expectations and degree of safety / fear of patients on returning to the hospital. Methods: Self-completion of an anonymous survey for patients over 12 years in the first 5 weeks of SCITA restart. CARAT was used as an instrument to assess rhinitis and asthma control. Results: 77 patients ( $90 \%$ adults, $68 \%$ women) were included, all with rhinitis and $40 \%$ with asthma. The average time interval between the last administration and the restart of SCITA was $13 \pm 2.48$ weeks. Seven percent of patients were seen in an emergency / unscheduled appointment due to the exacerbation of respiratory symptoms and only I had COVID-19. CARAT-Total scores showed control of rhinitis and asthma, respectively, in 35\% and $66 \%$ patients. The proportion of uncontrolled patients rised with the increase in the SCITA interruption period (IP). The majority (> 90\%) of patients reported no significant impact on their quality of life and maintained the usual control medication (48\%), expressed concern about losing the benefits of SCITA with its interruption (62\%) and reported feeling safer in the vaccination offices compared to the hospital building or waiting room. Conclusions: An increase in the frequency of uncontrolled patients with an increase in IP was observed. For most patients, the IP did not have a significant impact on quality of life. The frequency of patients who needed to be observed in an emergency / unscheduled appointment was low and only I reported having had COVID-19. The perception of safety in the vaccination room is higher than in the hospital's common spaces.

Keywords: Asthma, CARAT, COVID-19, medication, quality of life, rhinitis, subcutaneous immunotherapy with aeroallergen, symptoms.

(C) 202I Sociedade Portuguesa de Alergologia e Imunologia Clínica. Published by Publicações Ciência \& Vida. This is an open access article under the CC BY-NC-ND license (http://creativecommons.org/licenses/by-nc-nd/4.0/).

\section{INTRODUÇÃO}

s primeiros casos de COVID-19 (Corona Vírus Disease 2019) foram descritos no continente Asiático, mais propriamente na cidade de Wuhan, na China, em dezembro 2019. Propagou-se rapi- damente a todos os continentes, sendo que, a 31/07/2020 existem, a nível mundial, 17064064 infetados e 668073 mortes por COVID-191 e, em Portugal, 51.072 infetados e 1.735 mortes por COVID-192. A Organização Mundial de Saúde (OMS) declarou a infeção por SARS-CoV-2 (Severe Acute Respiratory Syndrome Coronavirus 2) como 
uma emergência de saúde pública internacional a 30 de janeiro 2020 e classificou-a como pandemia a II de março de $2020^{3}$. O primeiro caso diagnosticado em Portugal data de 2 de março de $2020^{4}$.

No contexto da pandemia COVID-19 foi necessário adotar, a vários níveis, procedimentos adequados à evolução das diferentes fases de propagação da pandemia de modo a salvaguardar a manutenção da saúde pública. Neste sentido, o Governo português decretou o estado de emergência (EE) nacional em 18 março ${ }^{5}$, posteriormente prolongado até 2 maio de 2020, por forma a promover o confinamento da população e mitigar o contágio por SARS-CoV-2.

Ao nível dos cuidados de saúde, foi necessário todas as especialidades médicas, médico-cirúrgicas e cirúrgicas, implementarem medidas dirigidas aos doentes com e sem COVID-19. Assim, foram adotadas medidas gerais, de acordo com as diretivas publicadas em documentos internacionais e em documentos nacionais da Direção-Geral de Saúde (DGS) ${ }^{6}$ e, ainda, medidas específicas a cada especialidade, seguindo as diretrizes que foram gradualmente emitidas pelos conselhos de administração dos vários hospitais e centros de saúde.

Neste contexto, durante os 45 dias do EE, a direção do Serviço de Imunoalergologia do nosso hospital, seguindo as recomendações do Colégio da Especialidade de Imunoalergologia ${ }^{7}$, implementou, com o apoio do conselho de administração hospitalar, algumas modificações na atividade assistencial hospitalar e na realização de técnicas diagnósticas e procedimentos terapêuticos. $\mathrm{Na}$ Unidade Funcional de Imunoterapia com Alergénios deu-se prioridade à administração de imunoterapia subcutânea a venenos de himenópteros, realizada em contexto de Hospital Dia de Imunoalergologia. A administração da imunoterapia subcutânea a aeroalergénios (ITASC), realizada habitualmente no espaço físico da Consulta Externa de Imunoalergologia, foi suspensa, tendo em conta as alterações instituídas às condições de funcionamento desse espaço, nomeadamente com deslocação da equipa de enfermagem para os espaços de atendimento a doen- tes com COVID-19. Terminado o EE, reiniciou-se a administração de ITASC com a adoção de medidas de prevenção COVID-19 preconizadas pela DGS, como agendamentos espaçados de 30 minutos para cada doente, adoção de medidas físicas para promover um maior distanciamento social e implementação de um sistema de triagem dos utentes à entrada da unidade.

A opção de retomar a ITASC foi ponderada de forma a manter o benefício desta terapêutica sem aumentar o risco de infeção COVID-19, como defendido pelas normas da AAAAI (American Academy of Allergy Asthma \& Immunology), da WAO (World Allergy Organization) ${ }^{9}$ e da ARIA/EAACI (Allergic Rhinitis and its Impact on Asthma/European Academy Allergy Clinical Immunology) ${ }^{10,11}$, recentemente publicadas. Estas normas apelam à importância de, em tempo de pandemia, manter o controlo das doenças alérgicas através das três vertentes do tratamento da doença alérgica: evicção alergénica, terapêutica farmacológica de controlo e imunoterapia com alergénios. Nesses documentos é feita a sistematização das medidas gerais de prevenção de COVID-19 adaptadas à especialidade e de medidas específicas para ITASC, como, por exemplo, a contraindicação da administração em doentes infetados com COVID-19 que estejam em quarentena ou que estiveram em contacto com doentes infetados nos últimos 14 dias; o aumento do intervalo entra as administrações na ITASC, quer na fase indução quer de manutenção, sem colocar em causa a sua eficácia, sugerindo-se uma eventual opção pela via sublingual ${ }^{8-12}$.

O objetivo principal deste trabalho consiste em conhecer o impacto das medidas de confinamento no controlo dos doentes sob ITASC, percebendo se a interrupção da administração durante o período do EE da pandemia se associou ao recurso a consultas não programadas, a aumento da medicação de controlo e/ou alteração da qualidade de vida. Como objetivo secundário, pretendeu-se ainda conhecer os receios/preocupações dos doentes relativamente à interferência que esta suspensão poderá ter na sua doença e, ainda, o grau de segurança relativo ao regresso das administrações de ITASC em contexto hospitalar. 


\section{MATERIAIS E MÉTODOS}

\section{Estudo: Caracterização e contextualização}

Estudo observacional, transversal, recorrendo a inquérito de autopreenchimento, aplicado aos doentes que suspenderam a ITASC durante o período do EE.

A suspensão da ITASC foi comunicada a todos os doentes no início do EE por um Imunoalergologista em contexto de teleconsulta. Nesta, foram prestados aos doentes os esclarecimentos necessários sobre a sua patologia alérgica, a necessidade de manter terapêutica de controlo e foi feito o reforço das medidas de prevenção da COVID-19. Assim que a administração de ITASC foi retomada, os doentes foram contactados e a sua administração reagendada. O recomeço obedeceu a um esquema de reintrodução definido no serviço, com ajustes de doses de acordo com o intervalo de tempo em que a ITASC esteve suspensa, até se atingir a dose de manutenção definida como a dose eficaz para aquele doente e vacina.

\section{População-alvo}

Doentes com alergia respiratória previamente diagnosticada por imunoalergologista em programa de administração de ITASC na consulta externa de Imunoalergologia e a quem, atendendo ao plano de contingência hospitalar durante o EE, foi interrompida a administração de ITASC. O período de suspensão teve início em 18 de março e terminou a 2 de maio de 2020. Todos os doentes com mais de 12 anos, no dia em que retomaram a administração de ITASC, foram convidados a preencher este inquérito por um elemento da equipa de enfermagem. Os doentes que aceitaram preencher o inquérito deram o seu consentimento. $O$ inquérito esteve disponível para preenchimento nas primeiras cinco semanas após o reinício da administração de ITASC na Unidade Funcional de Imunoterapia com Alergénios do nosso serviço.

\section{Recolha de dados por inquérito}

Inquérito anónimo (Anexo I) desenhado para autopreenchimento exclusivamente pelo doente.
O inquérito era constituído por três partes: na primeira parte pretendeu-se conhecer alguns dados demográficos, clínicos e a ITASC de cada doente; na segunda avaliou-se se o período da interrupção da administração de ITASC se associou ou não a aumento do consumo de medicação de controlo ou a recurso a consulta urgente/ Inão programada ou a internamento por agudização da patologia respiratória; a terceira teve como objetivo conhecer as expectativas/preocupações dos doentes relativamente ao impacto que esta suspensão poderá ter tido na sua doença, bem como analisar a sua perceção de segurança com o regresso ao hospital para administração de ITASC. Sendo este um inquérito anónimo para autopreenchimento, não foi consultado o processo clínico do doente e, por conseguinte, toda a informação clínica do doente foi autorreportada pelo mesmo.

\section{Avaliação do controlo da doença alérgica respiratória}

O CARAT (Teste de controlo da asma e rinite alérgica) é um questionário validado que, na prática clínica e de acordo com as recomendações do ARIA (Allergic rhinitis and its impact on asthma), permite avaliar simultaneamente $\circ$ grau de controlo da asma e rinite alérgicas ${ }^{13,14}$. Este questionário é composto por 10 perguntas e encontra-se disponível online no endereço www.caratnetwork.org. Nas primeiras 4 questões avalia-se o controlo dos sintomas das vias áreas superiores (CARAT-S) e nas restantes o controlo dos sintomas das vias aéreas inferiores (CARAT-I). É pedido ao doente que considere os sintomas apresentados nas últimas 4 semanas anteriores ao preenchimento do inquérito. A pontuação máxima possível nas primeiras 4 questões é de 12 e para as últimas 6 questões de 18 . A pontuação máxima possível para o CARAT é de 30. Pontuações totais (CARAT-Total) >24 indicam bom controlo global da doença alérgica respiratória; enquanto pontuações $>8$ no somatório dos itens I-4 estão associados a bom controlo dos sintomas das vias aéreas superiores e pontuações $\geq 16$ no somatório dos itens $5-10$ estão associados a bom controlo relativo às vias aéreas inferiores ${ }^{13-16}$. 


\section{Análise estatística}

Os dados foram recolhidos de forma totalmente anónima. A análise estatística dos resultados foi efetuada com recurso ao software SPSS 26.0 for Windows ${ }^{\circledR}$ (SPSS Inc., Chicago, EUA). Foi realizada a análise descritiva dos dados através de frequências absolutas, média, desvio-padrão (DP), valor mínimo e máximo. Para as pontuações CARAT-S, CARAT-I e CARAT-Total foi indicado valor da mediana e intervalo interquartil. Os valores das pontuações CARAT (pontuação total e parciais) foram comparadas quanto ao sexo, idade e patologia utilizando o teste $t$ de Student. As diferenças entre estas pontuações relativamente ao início da ITASC ou duração da suspensão da ITASC foram testadas através do teste Kruskal-Wallis. Foram considerados como significativos valores de $\mathrm{p}<0,05$.

\section{RESULTADOS}

Durante as 5 semanas em que o inquérito foi aplicado, estavam programadas 142 administrações de ITASC. A estas 30 doentes não compareceram, 27 recusaram preencher o inquérito, e não foram incluídas no estudo 8 crianças com idade $\leq 12$ anos que realizaram vacina

Quadro I. Características demográficas, clínicas e da imunoterapia da população

\begin{tabular}{|c|c|c|c|}
\hline \multirow{2}{*}{ Doentes incluídos } & \multirow{2}{*}{$\frac{n(\%)}{77(100)}$} & \multicolumn{2}{|c|}{ Clínica referida - n (\%) } \\
\hline & & Rinite & Rinite + asma \\
\hline \multicolumn{4}{|l|}{ Sexo } \\
\hline $\begin{array}{l}\text { Feminino } \\
\text { Masculino }\end{array}$ & $\begin{array}{l}52(68) \\
25(32)\end{array}$ & $\begin{array}{l}31(67,4) \\
15(32,6)\end{array}$ & $\begin{array}{l}21(67,7) \\
10(32,2)\end{array}$ \\
\hline Idade (anos) & 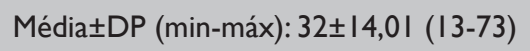 & - & - \\
\hline $\begin{array}{l}\text { Adultos } \\
\text { Adolescentes (>12 anos) }\end{array}$ & $\begin{array}{l}69(90) \\
8(10)\end{array}$ & $\begin{array}{c}42(91,3) \\
4(8,7)\end{array}$ & $\begin{array}{c}27(87,1) \\
4(12,9)\end{array}$ \\
\hline \multicolumn{4}{|l|}{ Idade - grupo etário (anos) } \\
\hline $\begin{array}{l}{[13-17]} \\
{[18-35]} \\
{[36-55]} \\
\geq 56\end{array}$ & $\begin{array}{c}8(10) \\
42(55) \\
22(29) \\
5(6)\end{array}$ & $\begin{array}{c}4(8,7) \\
29(63) \\
11(23,9) \\
2(4,3)\end{array}$ & $\begin{array}{c}4(\mid 2,9) \\
\text { I3 }(4 \mid, 9) \\
\text { II }(35,5) \\
3(9,7)\end{array}$ \\
\hline \multicolumn{4}{|l|}{ Composição da vacina } \\
\hline $\begin{array}{l}\text { Ácaros } \\
\text { Pólenes } \\
\text { Ácaros + pólenes } \\
\text { Fungos (Alternaria alternata) } \\
\text { Epitélios de animais e outros }\end{array}$ & $\begin{array}{l}36(4 \mathrm{I}) \\
17(22) \\
23(30) \\
I(1) \\
0(0)\end{array}$ & $\begin{array}{l}19(4 \mid, 3) \\
14(30,4) \\
12(16,1) \\
\text { I }(2,2) \\
0(0)\end{array}$ & $\begin{aligned} \text { I } & (54,8) \\
3 & (9,7) \\
\text { I I } & (35,5) \\
0 & (0) \\
0 & (0)\end{aligned}$ \\
\hline \multicolumn{4}{|l|}{ Ano de início da ITASC } \\
\hline $\begin{array}{l}2015 \\
2016 \\
2017 \\
2018 \\
2019 \\
2020\end{array}$ & $\begin{array}{c}6(8) \\
7(9) \\
10(13) \\
17(22) \\
33(43) \\
4(5)\end{array}$ & $\begin{array}{c}4(8,7) \\
7(15,2) \\
5(10,9) \\
8(17,4) \\
19(41,3) \\
3(6,5)\end{array}$ & $\begin{array}{c}2(6,5) \\
0(0) \\
5(16, I) \\
9(29) \\
14(45,2) \\
1(3,2)\end{array}$ \\
\hline
\end{tabular}

DP - Desvio-padrão; ITASC - Imunoterapia com aeroalergénios por via subcutânea 
nesse período. Incluíram-se, assim, 77 doentes que aceitaram preencher o inquérito.

Dos 77 doentes (idade média $\pm D P$ de $32 \pm \mid 4,01$ anos), $68 \%$ eram do sexo feminino e $90 \%$ adultos. Todos os doentes que responderam ao inquérito referiram ter o diagnóstico de rinite e 31 (40\%) destes referiam asma concomitante. Nove doentes (II,7\%) reportaram o diagnóstico de conjuntivite alérgica. A caracterização demo- gráfica e clínica dos participantes, bem como da imunoterapia realizada, está indicada no Quadro I. Não se verificaram diferenças estatisticamente significativas entre a duração ou o tipo de ITASC entre os doentes que reportaram ter apenas rinite e os que referiram ter rinite e asma ( $p=0,2$ e $p=0,13$, respetivamente).

O intervalo de tempo entre a última administração da ITASC antes do EE e o reinício da administração de

Quadro 2. Parâmetros avaliados durante o período de suspensão da vacina

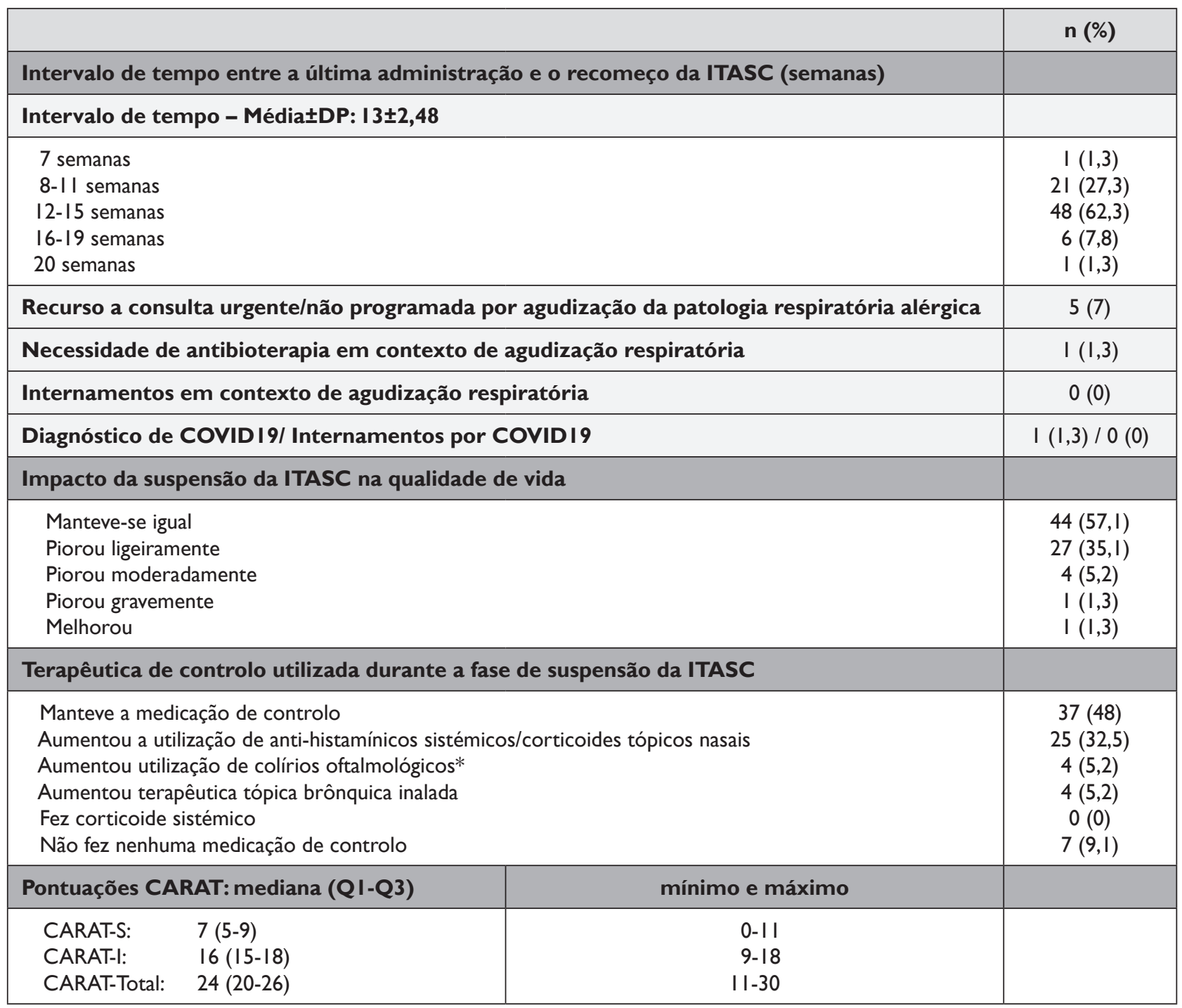

* Anti-histamínicos/estabilizadores da membrana dos mastócitos tópicos oculares; DP - Desvio-padrão; ITASC - Imunoterapia com aeroalergénios por via subcutânea; CARAT - Teste de controlo da asma e rinite alérgica; QI - primeiro quartil; Q3 - terceiro quartil 
ITASC depois deste período foi muito variável, tendo um tempo médio \pm DP de 13 $\pm 2,48$ semanas (mínimo: 7 semanas; máximo: 20 semanas) - Quadro 2 .

Durante o período de suspensão da ITASC, 5 (7\%) doentes referiram que necessitaram de ser observados em contexto de consulta urgente/não programada por agudização da sua doença respiratória alérgica e apenas I referiu ter tido o diagnóstico de COVID-19 - Quadro 2. Este doente não referiu ter diagnóstico de asma, apenas rinite alérgica, e não necessitou internamento. Não se verificou nenhuma associação entre o intervalo de tempo em que foi suspensa a ITASC e o recurso a consultas urgentes/não programadas $(p=0,69)$.

Relativamente à questão sobre a interferência dos sintomas de rinite, conjuntivite e/ou asma sobre a qualidade de vida, a maioria (93,5\%) dos doentes não referiu impacto significativo - Quadro 2. Ainda neste quadro estão indicadas as terapêuticas de controlo utilizadas durante o período de suspensão da ITASC. O único doente que referiu que a sua qualidade de vida melhorou também referiu que não fez nenhuma medicação durante o período de suspensão. No grupo dos doentes que

Quadro 3. Pontuações do CARAT estratificadas por sexo, grupo etário, patologia, duração total e da suspensão da ITASC imunoterapia subcutânea a aeroalergénios

\begin{tabular}{|c|c|c|c|c|c|c|c|c|c|}
\hline & \multicolumn{2}{|c|}{ CARAT-S } & \multirow[b]{2}{*}{$\mathbf{p}$} & \multicolumn{2}{|c|}{ CARAT-I } & \multirow[b]{2}{*}{$\mathbf{p}$} & \multicolumn{2}{|c|}{ CARAT-Total } & \multirow[b]{2}{*}{$\mathbf{p}$} \\
\hline & $\begin{array}{c}\text { Controlados } \\
\text { n (\%) }\end{array}$ & $\begin{array}{c}\text { Não } \\
\text { controlados } \\
\text { n (\%) }\end{array}$ & & $\begin{array}{c}\text { Controlados } \\
\text { n (\%) }\end{array}$ & $\begin{array}{c}\text { Não } \\
\text { controlados } \\
n(\%)\end{array}$ & & $\begin{array}{c}\text { Controlados } \\
n(\%)\end{array}$ & $\begin{array}{c}\text { Não } \\
\text { controlados } \\
\mathbf{n}(\%)\end{array}$ & \\
\hline \multicolumn{10}{|l|}{ Sexo } \\
\hline $\begin{array}{l}\text { Masculino } \\
\text { Feminino }\end{array}$ & $\begin{array}{c}8(32) \\
19(36,5)\end{array}$ & $\begin{array}{c}17(68) \\
33(63,5)\end{array}$ & 0,22 & $\begin{array}{c}20(80) \\
31(59,6)\end{array}$ & $\begin{array}{c}5(20) \\
21(40,4)\end{array}$ & 0,03 & $\begin{array}{c}12(48) \\
20(38,5)\end{array}$ & $\begin{array}{c}13(52) \\
32(61,5)\end{array}$ & 0,9 \\
\hline \multicolumn{10}{|l|}{ Grupo etário } \\
\hline $\begin{array}{l}\text { Adultos } \\
\text { Adolescentes ( }>12 \text { anos) }\end{array}$ & $\begin{array}{l}22(31,9) \\
5(62,5)\end{array}$ & $\begin{array}{l}47(68, I) \\
3(37,5)\end{array}$ & 0,03 & $\begin{array}{c}45(65,2) \\
6(75)\end{array}$ & $\begin{array}{c}24(34,8) \\
2(25)\end{array}$ & 0,18 & $\begin{array}{c}27(39,1) \\
5(62,5)\end{array}$ & $\begin{array}{c}42(60,9) \\
3(37,5)\end{array}$ & 0,08 \\
\hline \multicolumn{10}{|l|}{ Patologia reportada } \\
\hline $\begin{array}{l}\text { Rinite sem asma } \\
\text { Rinite com asma }\end{array}$ & $\begin{array}{l}12(26, I) \\
15(48,4)\end{array}$ & $\begin{array}{l}34(73,9) \\
16(51,6)\end{array}$ & 0,03 & $\begin{array}{l}31(67,4) \\
20(64,5)\end{array}$ & $\begin{array}{l}\text { I5 }(32,6) \\
\text { II }(35,5)\end{array}$ & 0,65 & $\begin{array}{l}16(34,8) \\
16(51,6)\end{array}$ & $\begin{array}{l}30(65,2) \\
15(48,4)\end{array}$ & 0,18 \\
\hline \multicolumn{10}{|l|}{ Início da ITASC } \\
\hline $\begin{array}{l}<\mathrm{I} \text { ano } \\
\text { I ano } \\
2 \text { anos } \\
3 \text { anos } \\
>3 \text { anos }\end{array}$ & $\begin{array}{c}\text { I }(25) \\
12(36,4) \\
6(35,3) \\
4(40) \\
4(30,8)\end{array}$ & $\begin{array}{c}3(75) \\
21(63) \\
11(64,7) \\
6(60) \\
9(62,2)\end{array}$ & 0,9 & $\begin{array}{c}3(75) \\
22(66,7) \\
10(58,8) \\
7(70) \\
9(69,2)\end{array}$ & $\begin{array}{c}\text { I }(25) \\
\text { II }(33,3) \\
7(4 I, 2) \\
3(30) \\
4(30,8)\end{array}$ & 0,9 & $\begin{array}{c}\text { I }(25) \\
\text { I5 }(45,5) \\
5(29,4) \\
6(60) \\
5(38,5)\end{array}$ & $\begin{array}{c}3(75) \\
18(54,5) \\
12(70,6) \\
4(40) \\
8(61,5)\end{array}$ & 0,9 \\
\hline \multicolumn{10}{|l|}{$\Delta t *$ suspensão ITASC } \\
\hline $\begin{array}{l}7 \text { semanas } \\
8-11 \text { semanas } \\
12-15 \text { semanas } \\
16-19 \text { semanas } \\
\geq 20 \text { semanas }\end{array}$ & $\begin{array}{c}1(100) \\
10(47,6) \\
16(33,3) \\
0(0) \\
0(0)\end{array}$ & $\begin{array}{c}0(0) \\
11(52,4) \\
32(66,7) \\
6(100) \\
1(100)\end{array}$ & 0,02 & $\begin{array}{c}\text { I }(100) \\
\text { I7 }(8 \mid) \\
3 \mid(64,6) \\
I(16,7) \\
0(0)\end{array}$ & $\begin{array}{c}0(0) \\
4(19) \\
17(34,4) \\
5(83,3) \\
1(100)\end{array}$ & 0,02 & $\begin{array}{c}\text { I }(100) \\
\text { II }(52,4) \\
\text { I9 }(39,6) \\
\text { I }(16,7) \\
0(0)\end{array}$ & $\begin{array}{c}0(0) \\
10(47,6) \\
29(60,4) \\
5(83,3) \\
1(100)\end{array}$ & 0,06 \\
\hline
\end{tabular}

* $\Delta \mathrm{t}$ - intervalo de tempo; CARAT - Teste de controlo da asma e rinite alérgica; CARAT-S - pontuação CARAT para as questões I a 4; CARAT-I - pontuação CARAT para as questões 5 a I0; CARAT-Total - pontuação total do CARAT 
Joana Cosme, Amélia Spínola Santos, Anabela Resende, Susana Marques, Elisa Pedro

referiram que a sua qualidade de vida se manteve idêntica, a maioria $(63,6 \%)$ manteve a medicação de controlo, enquanto no grupo dos doentes que referiram que a qualidade de vida piorou, a maioria (75\%) afirmou que teve necessidade de aumentar a utilização de medicação $(\mathrm{p}<0,05)$.

No Quadro 2 estão indicadas as pontuações medianas, máximas e mínimas do CARAT-S, CARAT-I e CARAT-Total.

No Quadro 3 indicam-se as pontuações do CARAT-S, CARAT-I e CARAT-Total estratificadas por sexo, grupo etário, patologia, tempo da ITASC e período de suspensão da ITASC. Considerando as pontuações do CARAT (Quadro 3) à data do preenchimento do inquérito, 27 (35\%) doentes apresentavam um bom controlo dos sintomas das vias áreas superiores e 51 (66\%) um bom controlo dos sintomas das vias áreas inferiores, mas só $32(42 \%)$ apresentavam uma pontuação CARAT-Total indicadora de bom controlo global. A percentagem de homens $(80 \%)$ com sintomas das vias aéreas inferiores controlados foi superior à encontrada nas mulheres $(59,6 \% ; p=0,03)$, e no grupo dos adolescentes a percentagem de doentes com controlo dos sintomas das vias aéreas superiores $(62,5 \%)$ foi superior à en-

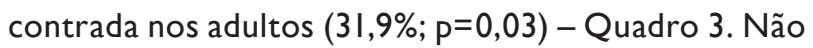
se verificaram diferenças significativas entre as pontuações CARAT e o tempo de duração da ITASC $(p=0,9)$. Verifica-se, ainda, que a proporção de doentes não controlados face aos controlados aumentou à medida que aumentou, também, o período de suspensão da ITASC, se se considerar o CARAT-S ou o CARAT-I $(p=0,02)$. Para o CARAT-Total as diferenças encontradas não foram estatisticamente significativas $(p=0,06)$. Não se verificaram diferenças significativas entre a composição da ITASC realizada e o grau de controlo sintomático $(p=0,54)$.

Relativamente à questão sobre as expectativas/preocupações dos doentes sobre a suspensão da ITASC durante $\circ \mathrm{EE}$, as respostas foram muito diversas e estão indicadas na Figura I.

$\mathrm{Na}$ Figura 2 estão indicadas as respostas dos doentes relativamente à sensação de segurança em cada um dos

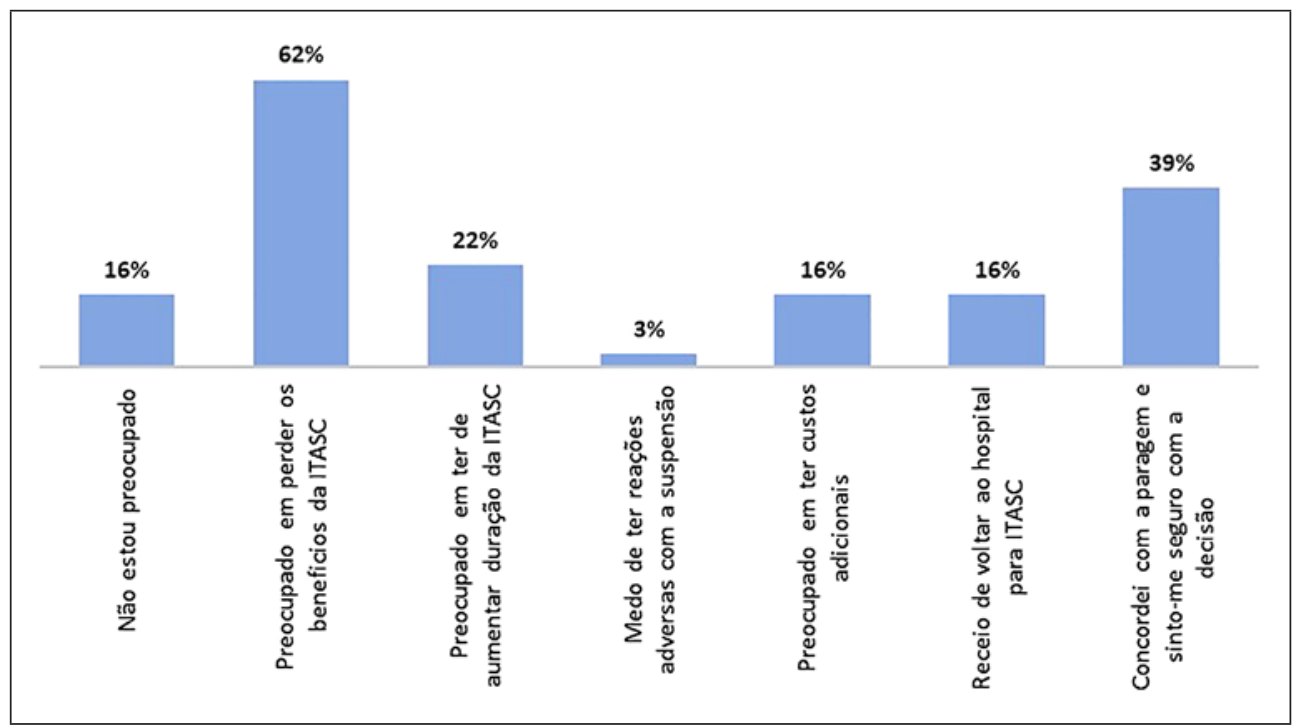

ITASC - Imunoterapia com alergénios por via subcutânea. Nota: Permitida mais do que uma resposta à questão.

Figura I. Preocupações/expetativas dos doentes com a suspensão da ITASC 


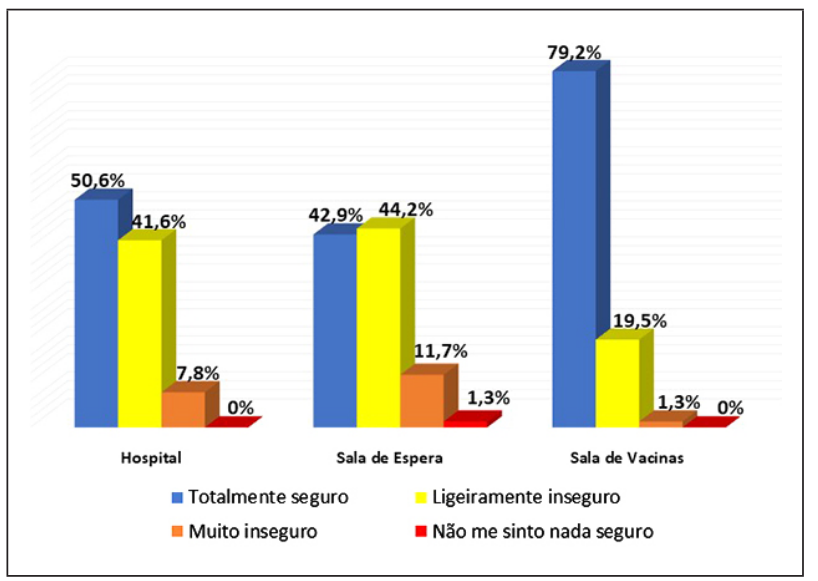

Figura 2. Perceção do grau de segurança nos diferentes espaços hospitalares

espaços que percorreram para poderem realizar a ITASC (edifício do hospital, sala de espera, sala de vacinas).

\section{DISCUSSÃo}

Responderam ao inquérito 77 doentes que habitualmente realizam ITASC no nosso serviço e que, durante - $\mathrm{EE}$, suspenderam a administração regular de vacina; sendo que todos autorreportaram rinite alérgica e $40 \%$ asma concomitante.

Neste estudo, no grupo dos doentes que reportam ter rinite e asma, $48,4 \%$ apresentaram um valor de CARAT-Total indicador de controlo sintomático. Estes dados diferem dos publicados por Pereira et al. ${ }^{15}$, que numa amostra de doentes adultos com diagnóstico médico de rinite e asma seguidos em consulta hospitalar de Imunoalergologia, documentaram que apenas $18,6 \%$ dos doentes tinham uma pontuação CARAT-Total indicadora de um bom controlo sintomático; e, ainda, dos publicados noutro estudo ${ }^{16}$ que identificaram apenas $14 \%$ dos doentes com rinite e asma referenciados a uma consulta de Imunoalergologia, com pontuações CARAT-Total indicadoras de controlo sintomático. Nestes estudos ${ }^{15,16}$, não surge discriminada a frequência de doentes sob imunoterapia com aeroalergénios.
Adicionalmente, no presente estudo, a proporção de doentes não controlados aumentou à medida que aumentou o período de suspensão da ITASC. Tais dados podem sugerir a existência de um maior controlo nos doentes que interromperam a ITASC durante menos tempo. Branco Ferreira et al. ${ }^{17}$ tinham já reportado que doentes com rinite e asma, submetidos a imunoterapia com alergénios tinham significativamente maior redução da gravidade clínica da asma e rinite e redução da medicação de controlo, quando comparados com doentes que não tinham sido submetidos a imunoterapia, e que este efeito benéfico era superior no final do segundo e terceiro anos de tratamento. Desta forma, pensamos que as diferenças encontradas no presente estudo também podem corroborar o efeito da imunoterapia com alergénios, em particular de uma ITASC com maior duração, na redução e controlo sintomático, desde que aplicada em doentes seleciona$\operatorname{dos}^{18-20}$, e mesmo em contexto de atraso/interrupção do esquema posológico.

Um aspeto que é digno de nota é o facto de, neste estudo, os doentes com rinite e asma terem um pior controlo dos sintomas de rinite ( $51,6 \%$ não controlados) do que dos sintomas de asma ( $35,5 \%$ não controlados). Embora os dados deste trabalho não nos permitam encontrar uma explicação para estas diferenças, pensamos que as mesmas se possam dever ao facto de tendencialmente ser prescrito mais frequentemente imunoterapia com alergénios aos doentes com formas ligeiras ou moderadas de asma do que aos doentes com asma grave. Contudo, estas justificações são meramente especulativas e não podem ser confirmadas com os resultados deste estudo.

Relativamente aos doentes que neste estudo autorreportaram rinite e que não referiram ter asma, verificamos que a percentagem de doentes com valores CARAT-S indicadores de controlo sintomático $(26,1 \%)$ é inferior à encontrada nos doentes com rinite e asma (48,4\% controlados). Tal diferença pode eventualmente justificar-se pela existência de distintas sensibilizações 
predominantes. O período de suspensão da ITASC decorreu entre 18 de março e 2 de maio de 2020, isto é, coincidiu com os meses de primavera, o que EE poderá ter reduzido a exposição a alergénios outdoor. Neste trabalho, quando se considera a percentagem de doentes que referiram estar a fazer imunoterapia a pólenes, verificamos que $56,5 \%$ dos doentes com rinite e sem asma estavam a fazer ITASC a pólenes, enquanto no grupo dos doentes com rinite e asma este valor era inferior $(45,2 \%)$. Contudo, as diferenças encontradas não foram estatisticamente significativas, pelo que esta explicação é meramente especulativa, não podendo ser demonstrada neste trabalho.

No grupo de doentes que refere ter rinite e não refere ter asma, seria de esperar que a percentagem com pontuações de CARAT-I reveladoras de não controlo fosse baixa. Assim, outro dado a sublinhar neste estudo é o facto de existirem cerca de $33 \%$ dos doentes que referiram ter rinite mas não referiram ter asma e apresentaram scores CARAT-I que os classificavam como não controlados relativamente às vias aéreas inferiores. Múltiplos trabalhos têm demonstrado, ao longo dos anos, que a disfunção das vias aéreas superiores e inferiores ocorre, com frequência, em conjunto e parece compartilhar fatores de risco comuns, como a atopia ${ }^{21}$; aproximadamente $30 \%$ dos doentes com rinite apresentam sintomas de asma e muitos asmáticos também apresentam sintomas de rinite ${ }^{22,23}$. Adicionalmente, cerca de $40 \%$ dos doentes com rinite alérgica sem nenhuma evidência clínica de asma apresentaram hiperreatividade brônquica identificada por uma prova de broncoprovocação com metacolina ${ }^{24}$. Assim, a percentagem de $33 \%$ referida é mais uma chamada de atenção para a existência, muitas vezes, de uma subvalorização, subdiagnóstico e subtratamento dos sintomas das vias aéreas inferiores nos doentes com rinite, tornando sempre mandatória a investigação aprofundada de queixas dos dois andares respiratórios em doentes que aparentemente só apresentam queixas de um deles ${ }^{22,24}$. Outro aspeto que merece ser res- salvado é que estes diagnósticos foram autorreportados pelos doentes, sem que os autores os tenham confrontado com a informação constante dos seus processos clínicos. Tal facto pode ainda sugerir que os doentes, muitas vezes, desconhecem o seu diagnóstico de asma, ou desvalorizam um diagnóstico de asma ligeira/intermitente e, assim sendo, não o incluíram nas suas respostas.

Verificamos que o número de doentes que necessitou de ser observado em contexto de consulta urgente/não programada foi reduzido, não se tendo registado internamentos por agudização da patologia respiratória. Um único doente referiu ter tido COVID-19, sem necessitar de internamento. Apesar de a presente amostra ser reduzida e, por isso, sem tirar conclusões, podemos especular que este aspeto está de acordo com trabalhos internacionais ${ }^{25,26}$ que mostram que os asmáticos, em particular com inflamação Th2, não parecem ter risco aumentado de infeção COVID-19 ou de formas mais graves desta doença ${ }^{25}$.

Um aspeto inovador deste trabalho foi a averiguação dos receios/preocupações dos doentes relativamente à interferência que esta suspensão poderá ter na sua doença e/ou qualidade de vida e, ainda, o grau de segurança relativo ao regresso da ITASC em contexto hospitalar. A avaliação dos pontos de vista pessoais dos doentes sobre a(s) sua(s) doença(s) e tratamentos fornece informações úteis que permitem uma abordagem terapêutica mais personalizada. Adicionalmente, o papel central das perspetivas dos doentes é defendido pelo Grau de Recomendações, Avaliação, Desenvolvimento e Avaliação (GRADE), recentemente adotado pela OMS como diretriz para a qualidade, evidência e força das recomendações ${ }^{27}$.

Neste estudo, apenas 6,5\% dos doentes referiu que a sua qualidade de vida piorou significativamente. Até à data, não estão publicados estudos que avaliem a interferência que o confinamento teve na qualidade de vida dos doentes alérgicos. Contudo, um estudo italiano recentemente publicado ${ }^{28}$, embora em doentes com 
imunodeficiência primária de anticorpos, avaliou o impacto na qualidade de vida das medidas de confinamento, nomeadamente utilização de teleconsultas em vez de consultas presenciais e de tratamentos de autoadministração no domicílio, neste caso com imunoglobulina subcutânea. Nesse estudo ${ }^{28}$, não se registaram diferenças na qualidade de vida entre os doentes que já faziam reposição de imunoglobulina no domicílio e aqueles que tiveram de iniciar esta reposição em casa devido à pandemia COVID-19. Estes autores ${ }^{28}$ objetivaram um risco de ansiedade/depressão associado à pandemia e não às condições clínicas relacionadas com a sua imunodeficiência, tendo grande parte dos doentes referido estar preocupado com a possibilidade de ter falta de medicamentos durante a pandemia. No nosso estudo, a maioria dos doentes referiu que a principal preocupação na suspensão da ITASC durante o EE foi o de perder os benefícios da ITASC e apenas 16\% referiram ter receio de voltar ao hospital para fazer $\circ$ tratamento.

No que se refere à questão sobre o grau de segurança nos diferentes espaços do hospital, cerca de $80 \%$ sentia-se seguro na sala de vacinas, enquanto apenas $43 \%$ se sentia seguro na sala de espera comum ao centro de ambulatório do hospital. Embora o conceito de segurança num espaço possa ser influenciado por múltiplas variáveis, podemos pensar que as medidas que adotamos, nomeadamente a promoção do distanciamento social, o reagendamento das ITASC, com maior intervalo entre doentes, e o sistema de triagem implementado na Unidade Funcional de Imunoterapia possam ter contribuído para que os doentes se sentissem mais seguros na sala de vacinas. As implementações destas medidas de prevenção da infeção COVID-19 devem, por isso, ser consideradas em estratégias futuras, nomeadamente na promoção de espaços próprios e individualizados para cada especialidade, em vez de espaços grandes e de utilização comum $6,10,11$.

No presente trabalho identificaram-se $21 \%$ de faltas ao reagendamento da ITASC após o período de suspen- são (30 faltas em 142 doentes). No ano de 2019, no nosso serviço, em igual período registaram-se apenas $15 \%$ de faltas à ITASC. A frequência de não adesão à ITASC varia entre 6 e $84 \%{ }^{29}$ e é influenciada por diversos fatores como a existência de reações adversas, os custos associados ou a duração da ITASC ${ }^{29,30}$. A suspensão temporária da ITASC, que neste estudo se associou a uma menor adesão, pode ser considerada um outro fator adicional para a não adesão, podendo contribuir para a descontinuação do tratamento de alguns doentes.

Este estudo tem as limitações decorrentes do facto de se tratar de um inquérito autopreenchido pelos doentes e, portanto, avaliar aspetos relativamente subjetivos e autoavaliados pelos próprios. No entanto, o objetivo era realizar um estudo da "vida real hospitalar" que avaliasse o impacto da interrupção da ITASC e conhecesse os receios dos doentes na retoma da ITASC em contexto hospitalar. Por ser um estudo totalmente anónimo, não tivemos acesso a dados clínicos anteriores de controlo destes doentes, sendo este aspeto limitador da obtenção de conclusões mais objetivas.

\section{CONCLUSÃO}

Em conclusão, durante o período de suspensão da ITASC o número de doentes com sintomas de alergia respiratória controlados diminuiu à medida que aumentou a duração da suspensão. Ainda sobre o controlo dos sintomas de doença alérgica, verificou-se que as vias respiratórias superiores estavam menos controladas do que as inferiores. Embora alguns doentes tenham referido que o período de suspensão da ITASC teve impacto significativo na sua qualidade de vida, a maioria $(>90 \%)$ não o referiu. A frequência de doentes que necessitou de ser observado em consulta urgente/não programada foi baixa e apenas I referiu ter tido COVID-19. A maioria dos doentes sentiu-se seguro em retomar a ITASC. Este grau de segurança foi maior na sala de vacinas, comparativamente com a sala de espera comum ou outros espaços 
comuns do hospital. Este último aspeto obriga a repensar o futuro redimensionamento do espaço físico partilhado com outras especialidades como medida de prevenção de COVID-19.

\section{Conflito de interesses}

Os autores declaram que não existem conflitos de interesses.

\section{ORCID:}

Joana Cosme (iD) 0000-0002-2144-3236

Amélia Spínola Santos (ID) 0000-0002-5032-6354

Elisa Pedro (iD) 0000-0002-1242-3524

\section{Contacto:}

Joana Cosme

Serviço de Imunoalergologia - Hospital de Santa Maria,

Av. Prof. Egas Moniz

1649-035 Lisboa

E-mail: joanamcosme@gmail.com

\section{REFERÊNCIAS}

I. Who.int [homepage on the Internet]. World health organizations - coronavirus disease (COVID-19) pandemic online resources; 2020 [cited 2020 July I]. Available from: https://www.who.int/ emergencies/diseases/novel-coronavirus-2019.

2. DGS.pt [homepage on the Internet]. Direção-Geral da Saúde COVIDI9; 2020 [cited 2020 July I]. Available from: https://covidI9. min-saude.pt/.

3. Who.int [homepage on the Internet]. Archived: WHO Timeline - COVID-19; 2020 [cited 2020 July I]. Available from: https://www. who.int/news-room/detail/27-04-2020-who-timeline-covid-19.

4. DGS.pt [homepage on the Internet]. CI60_75_vl DE 2/3/2020Casos de infeção por novo Coronavírus (COVIDI9); 2020 [cited 2020 June 20]. Available from: https://www.dgs.pt/a-direccao-geral-dasaude/comunicados-e-despachos-do-director-geral.aspx.

5. Decreto n. 2-A/2020 de 2020-03-20. Diário da República n. ${ }^{\circ}$ 57/2020, I. ${ }^{\circ}$ Suplemento, Série I. Presidência do Conselho de Ministros. Lisboa.

6. DGS.pt [homepage on the Internet]. Norma 01 I/2020 de 17/3/2020 - Medidas de prevenção da transmissão em estabelecimentos de atendimento ao público; 2020 [cited 2020 June 20]. Available from: https://www.dgs.pt/a-direccao-geral-da-saude/comunicados-edespachos-do-director-geral.aspx.
7. Ordemdosmedicos.pt [homepage on the internet]. Ordem dos Médicos - Colégio de Imunaolergologia. Restrição de atividades nas instituições de saúde e proteção individual. 2020 [cited 2020 June 19]. Available from: https://ordemdosmedicos.pt/recomendacoes sobre a restricao de atividades nas-instituicoes-de-saude-e-protecao-individual-em-imunoalergologia/.

8. Epstein T, Ponda P, Creticos P. Administration of subcutaneous allergen immunotherapy during the COVID-19 outbreak: a Work Group Report of the AAAAI Immunotherapy, Allergen Standardization and Allergy Diagnostics (IASAD) Committee 2020. 2020 [cited 2020 June 30]. Available from: https://education.aaaai.org/ immunotherapy_covid-19.

9. Who.int [homepage on the Internet]. World Allergy Organization Preparing your office for the COVID-19 pandemic. 2020 [cited 2020 June 19]. Available from: https: www.worldallergy.org/UserFiles/file/Preparing_your-office_COVID-19.pdf.

10. Pfaar O, Klimek L, Jutel M, Akdis C, Bousquet J, Akdis M, et al. Handling of allergen immunotherapy in the COVID-19 pandemic: An ARIA-EAACI statement. Allergy 2020;75:1546-54. doi: 10. IIII/ all. 14336.

II. Pfaar O, Klimek L, Jutel M, Akdis CA, Bousquet J, Breiteneder H et al. COVID-19 pandemic: Practical considerations on the organization of an allergy clinic - an EAACI/ARIA Position paper. Allergy 2021;76:648-676. doi: 10.1III/all.14453.

12. Nickels A, Gupta P, Tamesis G. COVID-19's impact on allergists' clinical services and protocols 2020. [cited 2020 June 30]. Available from: https://college.acaai.org/publications/college-insider/ covid-19s-impact-allergistsclinical-services-and-protocols.

13. Nogueira-Silva L, Martins SV, Cruz-Correia R, Azevedo LF, Morais-Almeida M, Bugalho-Almeida A, et al. Control of allergic rhinitis and asthma test - a formal approach to the development of a measuring tool. Respir Res 2009;10:52. doi: 10.1186/I465-992I-10-52

14. Fonseca JA, Nogueira -Silva L, Morais-Almeida M, Azevedo L, Sá -Sousa A, Branco-Ferreira M, et al. Validation of a questionnaire (CARATI0) to assess rhinitis and asthma in patients with asthma. Allergy 2010; 65:1042 -8. doi: 10.1 III/j.1398-9995.2009.02310.x

15. Pereira A, Martins C, Fonseca J. Utilização conjunta do CARAT e função respiratória na avaliação do controlo da asma e rinite. Rev Port Imunoalergologia 2013; 21:103-15.

16. Ricardo Pereira P, Lopes C. Estudo transversal da avaliação do controlo da rinite e asma alérgicas em consulta hospitalar de Imunoalergologia através do questionário CARATIO. Rev Port Pneumologia 2013; 19:163-67.

17. Branco Ferreira M, Rodrigues Alves R, Pereira Barbosa M. Imunoterapia específica: Uma mais-valia no tratamento da asma e rinite alérgicas. Rev Port Imunoalergologia 2009; 17: 13-35.

18. Spínola Santos A, Branco Ferreira M, Pereira Barbosa M. Imunoterapia específica e controlo da asma. Rev Port Imunoalergologia 2012; 20: 109-20. 
19. Dhami S, Kakourou A, Asamoah F, Agache I, Lau S, Jutel M, et al. Allergen immunotherapy for allergic asthma: A systematic review and meta-analysis. Allergy 2017;72:1825-48. doi: 10.1III/all.13208.

20. Gani F, Lombardi C, Barrocu L, Landi M, Ridolo E, Bugiani M, et al. The control of allergic rhinitis in real life: a multicenter cross-sectional Italian study. Clin Mol Allergy 2018;16:4. doi: 10.1/86/ s12948-018-0082-y.

21. Verdiani P, Di Carlo S, Baronti A. Different prevalence, and degree of non-specific bronchial hyperreactivity between seasonal and perennial rhinitis. J Allergy Clin Immunol 1990; 86:576-78. doi: 10.1016/s0091-6749(05)80215-5.

22. Shaaban R, Zureik M, Soussan D, Antó JM, Heinrich J, Janson C, et al. Allergic rhinitis and onset of bronchial hyperresponsiveness: a population-based study. Am J Respir Crit Care Med 2007; 176:659-66. doi: 10.1164/rccm.200703-427OC.

23. Cirillo I, Vizzaccaro A, Tosca MA, Negrini S, Negrini AC, Marseglia $G$, et al. Bronchial hyperreactivity and spirometric impairment in patients with allergic rhinitis. Monaldi Arch Chest Dis 2005; 63:79-83. doi: 10.408I/monaldi.2005.642.

24. S Braman S, A Barrows A, A DeCotiis B, A Settipane G, M Corrao W. Airway hyperresponsiveness in allergic rhinitis. A risk factor for asthma. Chest 1987; 91:67I-4. doi: 10.1378/ chest.91.5.67I.
25. Carli G, Cecchi L, Stebbing J, Parronchi P, Farsi A. Is asthma protective against COVID-19 Allergy 2021;76:866-8. doi: 10.1III/all.14426.

26. Morais-Almeida M, Pité H, Aguiar R, Ansotegui I, Bousquet J. Asthma and the coronavirus disease 2019 pandemic: A literature review. Int Arch Allergy Immunol 2020;|81:680-8. doi: 10.1159/ 1000509057.

27. Guyatt GH, Oxman AD, Vist GE, Kunz R, Falck-Ytter Y, Alonso-Coello $P$, et al. Rating quality of evidence and strength of recommendations GRADE: an emerging consensus on rating quality of evidence and strength of recommendations. BMJ 2008; 336:924-6. doi: I0.1136/bmj.39489.470347.AD.

28. Pulvirenti F, Cinetto F, Milito C, Bonanni L, Pesce AM, Leodori $G$, et al. Health-related quality of life in common variable immunodeficiency italian patients switched to remote assistance during the COVID-19 pandemic. J Allergy Clin Immunol Pract 2020;8:1894-9.e2. doi: 10.1016/j.jaip.2020.04.003.

29. Passalacqua G, Baiardini I, Senna G, Canonica GW. Adherence to pharmacological treatment and specific immunotherapy in allergic rhinitis. Clin ExpAllergy 20 I3;43:22-8. doi: I0. I I I //j. I365-2222.20 I2. 04052.x.

30. Roberts G, Pfaar O, Akdis CA, Ansotegui IJ, Durham SR, Gerth van Wijk R, et al. EAACI Guidelines on allergen immunotherapy: Allergic rhinoconjunctivitis. Allergy 2018;73:765-98. doi: 10.IIII/all.I33I7. 
Anexo I. Inquérito fornecido aos doentes sob ITASC, seguidos no Serviço de Imunoalergologia do CHULN

\section{INQUÉRITO AOS DOENTES SOB IMUNOTERAPIA SUBCUTÂNEA COM AEROALERGÉNIOS (VACINAS) SEGUIDOS NO SERVIÇO DE IMUNOALERGOLOGIA DO CHULN}

(A realizar aos doentes que recomeçaram vacina no tempo de espera/vigilância após a administração)

Caro utente do Serviço de Imunoalergologia,

Estamos a realizar um inquérito a todos os doentes que fazem vacinas antialérgicas injetáveis (administração subcutânea) no Serviço de Imunoalergologia do Hospital De Santa Maria (CHULN).

Durante o Estado de Emergência devido à pandemia COVID19 alguns procedimentos programados, como a administração de vacinas antialérgicas, tiveram de ser adiados.

O objetivo deste inquérito é perceber de que forma este adiamento poderá ter influenciado a qualidade de vida dos nossos doentes.

Os resultados deste inquérito permitirão perceber o grau de satisfação dos nossos doentes e melhorar as nossas práticas, por forma a prestarmos Serviços de elevada qualidade aos nossos doentes.

A sua opinião é, para nós, muito importante!

Pedíamos, assim, o favor de responder a este inquérito anónimo (Por favor, não coloque o seu nome em nenhum lugar do inquérito). A confidencialidade dos dados recolhidos será totalmente assegurada e os resultados obtidos serão utilizados para fins científicos e clínicos.

Muito obrigado pela sua colaboração

Por favor, depois de responder, entregue o inquérito à equipa de enfermagem. Não se esqueça! 


\section{INQUÉRITO AOS DOENTES A FAZER VACINA SUBCUTÂNEA COM AEROALERGÉNIOS}

Por favor, indique os seguintes dados:

Idade: (anos)

Sexo: Masculino Feminino

Data de preenchimento: / 2020

1) Relativamente às doenças pelas quais está a ser seguido na consulta de Imunoalergologia, por favor, escolha a opção que melhor caracteriza a sua situação (coloque uma cruz (X) à frente da opção correta):

a. Eu tenho rinite alérgica

b. Eu tenho asma alérgica

c. Eu tenho rinite e conjuntivite alérgicas

d. Eu tenho rinite e asma alérgicas

e. Eu tenho rinite, conjuntivite e asma alérgicas

2) Em que ano iniciou a vacina antialérgica (Indique o ano à frente):

3) A sua vacina contém que alergénios (coloque uma cruz (X) à frente da opção correta):
a. Ácaros
b. Pólenes
c. Ácaros e pólenes
d. Epitélios de animais
e. Fungos (Alternaria alternata)
f. Outra opção (Indique qual:

4. Em que data fez a última administração de vacina antialérgica (antes do início do estado de emergência devido à COVID19): $/ 2020$

5. Em relação aos meses de março e abril de $\mathbf{2 0 2 0}$ (quando suspendeu a administração da sua vacina antialérgica) responda às seguintes questões (coloque uma cruz $(X)$ à frente da opção correta):

5.1) Teve de ser observado em consulta de urgência/consulta não programada por agravamento rinite/sinusite/asma:

$\operatorname{Sim}$

Não

Página 2 de 5 
5.2) Teve necessidade de ser internado em algum Serviço de internamento hospitalar por agravamento rinite/sinusite/asma:

$\operatorname{Sim}$

Não

5.3) Teve necessidade de fazer antibiótico por infeção respiratória:

$\operatorname{sim}$

Não

5.4) Teve infeção por COVID 19:

$\operatorname{Sim}$

(Se Sim: Teve agravamento da sua asma? Sim Não

Não

6. Em relação às últimas 4 semanas (período em que teve suspensa a administração da sua vacina antialérgica) e relativamente aos seus sintomas de rinite/sinusite/asma quando parou a vacina indique (Faça uma cruz (X) na opção correta):

\begin{tabular}{|c|c|c|c|c|}
\hline & Nunca & Até 1 ou 2 dias & $\begin{array}{l}\text { Mais de } 2 \text { dias } \\
\text { por semana }\end{array}$ & $\begin{array}{l}\text { Quase todos ou } \\
\text { todos os dias }\end{array}$ \\
\hline 1. Nariz entupido? * & $\bigcirc$ & O & $\bigcirc$ & 0 \\
\hline 2. Espirros?* & O & O & O & O \\
\hline 3. Comichao no nariz? * & 0 & O & 0 & 0 \\
\hline 4. Corrimento/pingo do nariz? ${ }^{*}$ & O & $\bigcirc$ & O & 0 \\
\hline 5. Falta de ar/dispeneia? * & O & O & O & O \\
\hline 6. Chiadeira no peito/pieira? * & O & $\bigcirc$ & 0 & O \\
\hline 7. Aperto no peito com esforço físico? * & O & ○ & O & O \\
\hline $\begin{array}{l}\text { 8. Cansaço/dificuldade em fazer as suas actividades ou } \\
\text { tarefas do dia-a-dia? * }\end{array}$ & O & O & O & O \\
\hline $\begin{array}{l}\text { 9. Acordou durante a noite por causa da sua } \\
\text { asma/rinite/alergia? * }\end{array}$ & 0 & O & $\bigcirc$ & 0 \\
\hline 0. Aumentar a utilização dos seus medicamentos? * & 0 & $\bigcirc$ & O & 0 \\
\hline
\end{tabular}

Página 3 de 5 
7. Em relação aos meses de março e abril de $\mathbf{2 0 2 0}$ (quando suspendeu a administração da sua vacina antialérgica) e relativamente à utilização de medicação de controlo para a sua rinite/sinusite/asma/conjuntivite (escolha a opção correta):

a. Mantive a medicação que já fazia

b. Tive necessidade de aumentar a utilização de anti histamínicos e/ou spray nasais (corticoide nasal)

c. Tive necessidade de aumentar a utilização de colírios oculares antialérgicos

d. Tive necessidade de aumentar a utilização de inaladores brônquicos por crises de asma/dificuldade respiratória/tosse alérgica

e. Tive necessidade de fazer corticoide oral (comprimidos de cortisona)

f. Não fiz qualquer medicação

8. Em relação aos meses de março e abril de $\mathbf{2 0 2 0}$ (quando suspendeu a administração da sua vacina antialérgica) como classifica a interferência da sua rinite/sinusite/asma/conjuntivite na sua qualidade de vida (escolha a opção correta):

a. A minha qualidade de vida melhorou

b. A minha qualidade de vida manteve-se idêntica (não piorou)

c. A minha qualidade de vida piorou ligeiramente

d. A minha qualidade de vida piorou moderadamente

e. A minha qualidade de vida piorou gravemente

9. Relativamente à suspensão da vacina antialérgica nos meses de março e abril de 2020, gostaríamos de conhecer as suas expectativas/preocupações. Assim, escolha, por favor, até 2 opções dentro das frases indicadas abaixo que melhorem caracterizem a sua opinião:

a. Não estou preocupado(a) com a interrupção da vacina

b. Estou preocupado(a) em perder os efeitos benéficos da vacina com esta suspensão

c. Estou preocupado(a) por ter de eventualmente prolongar mais o tempo de administração da vacina

d. Tenho medo de ter reações adversas (dor/vermelhidão/hematoma/edema local) agora que reiniciei a vacina depois destes 2 meses de paragem

e. A questão económica preocupa-me já que posso ter de encomendar mais doses devido a esta paragem

f. Ainda tenho receio de vir ao hospital para nova administração

g. Concordei com esta paragem e sinto-me seguro(a) com esta decisão

10. Relativamente ao recomeço da administração de vacinas no Serviço de Imunoalergologia, gostaríamos de saber o quanto se sente seguro relativamente à sua presença nos seguintes locais: (Faça uma cruz na opção que melhor traduz a sua opinião)

10.1 No Hospital

a. Totalmente seguro(a)

b. Ligeiramente inseguro(a)

c. Muito inseguro(a)

d. Não me sinto nada seguro(a)

Página $\mathbf{4}$ de 5 
10.2 Na sala de espera das consultas externas/centro de ambulatório

a. Totalmente seguro(a)

b. Ligeiramente inseguro(a)

c. Muito inseguro(a)

d. Não me sinto nada seguro(a)

10.3 Na sala de enfermagem e espaço contíguo (espaço onde faz a vigilância após a vacina)

a. Totalmente seguro(a)

b. Ligeiramente inseguro(a)

c. Muito inseguro(a)

d. Não me sinto nada seguro(a)

\section{FIM DO QUESTIONÁRIO}

O Serviço de Imunoalergologia agradece o tempo dispensado para responder a este questionário. Muito obrigada pela sua colaboração!

A sua colaboração permitirá melhorar a qualidade dos Serviços prestados aos nossos doentes.

Por favor, depois de responder, entregue o inquérito à equipa de enfermagem. Não se esqueça!

Página 5 de 5 